\section{Definición de las}

\section{áreas y lineas de}

\section{investigación de la}

\author{
Facultad de
}

\section{Ingenieria de la}

Universidad

\section{Militar "Nueva}

Granada"
LUZ YOLANDA MORALES DE MOSQUERA, Ms.C. "*

\begin{abstract}
II 0 a Facultad de Ingeniería de la Universidad Militar tiene a disposición de las Fuerzas Militares y de la comunidad en general tres programas de Ingeniería en pregrado: Civil, Industrial y Mecatrónica; y dos en posgrado: Diseño, Construcción y Conservación de Puentes, y Gerencia Integral de Proyectos. Uno de los objetivos principales es desarrollar la investigación como característica fundamental de la docencia con el fin de generar conocimientos científicos y técnicos, tanto en programas de pregrado como de posgrado y como aporte a la solución de problemas de la comunidad mediante el servicio de extensión universitaria.
\end{abstract}

La definición de las Líneas de investigación de la Facultad se ha venido haciendo desde 1994, teniendo presente el recurso humano con que se cuenta y los recursos económicos con los que la Universidad apoya los proyectos de investigación, que cumplen con los requisitos establecidos en la resolución 458 del 28 de octubre de 1994 y que se enmarcan dentro de las políticas y estrategias de la Universidad en el campo de la investigación.

\footnotetext{
- Ingeniera Civil, MsC. Especialista en Proyectos de Desarrollo ESAP, Directora del Centro de investigaciones de la Facultad de Ingeniería de la Universidad Militar «Nueva Granada», Miembro de la Comisión de Enseñanza de la Sociedad Colombiana de Ingenieros.
} 
Otro aspecto que se ha tenido en cuenta es el desarrollo de Proyectos de investigación conjuntamence con entidades privadas o públicas, para fortalecer la relación Universidad - Entpresa, aunando esfuerzos, aprovechando recursos y compartiendo resultados y experiencias para lo cual la Universidad, cuenta con un grupo de profesionales e investigadores en los programas de lngeniería Civil, Industrial y Mecatrónica.

Antes de definir las líneas de Investigación fue necesario establecer los conceptos de área, línea y proyecto de investigación, los que se presentan a continuación:

AREA: Unidad temática del conocimiento, de carácter general. de la cual se derivan líneas de investigación.

LINEA: Conjunto de proyectos que busca resolver o avanzar en la solución de un problema dentro de чла determinada área. Tiende a resolver un problema macro, haciéndolo por etapas para definir en cada una de ellas un tópico específico o parcial, hasta lograr que la suma de los resultados obtenidos en estas etapas consoliden o aporten a la solución del problema planteado.

PROYECTO: Etapa de concepción, planeamienco y formulación de las acciones que permitirán lograr un objetivo general.

Partiendo de las áreas temáticas de los diferentes programas de Ingeniería, los recursos humanos, el soporte bibliográfico y técnico, al igual que los convenios y contactos con otras encidades públicas y privadas, durante los últimos cinco años, se han implementado las siguientes áreas y líneas de investigación: (ver Fig. 1)

\section{Area Institucional}

En el área Institucional se desarrollan los proyectos de investigación relacionados con la autoevaluación, currículo, plan de estudios, infraestructura, etc., para beneficio de la institución. En ella participan los directivos y docentes de la Facultad y se han desarrollado los siguientes proyectos:

- Autoevaluación de la Facultad de Ingeniería 1995

- Actualización del currículo de la Facultad 1998

- Actualización de los manuales de procedimiento y funciones de la Facultad. 1999, 2000

- Propuesta Técnica - económica para la dotación de los laboratorios de la Facultad

- Implementación de las pruebas de cross y de orificios para el laboratorio de hidráulica de la UMNG

Se proyecta establecer las líneas de investigación en Autoevaluación, curriculo e infraestructura.

\section{Area Básica Científica}

El área básica científica, está llamada a liderar la investigación en las demás áreas del saber en Ingeniería, dado su carácter básico sus resultados pueden extrapolarse a los diferentes aspectos e intereses de la facultad. Dentro de este marco conceptual, se ha propuesto participar en proyectos conjuntos con otros Centros e lnstitutos $\mathrm{co}$ lombianos para apoyar investigaciones que asi lo requieran y al mismo tiempo desarrollar e implementár, los nuevos conocimientos, que surgen en la comunidad académica internacional, incluyendo entre ellos los nuevos paradigmas científicos, que sin lugar a dudas cambiarán nuestra forma de pensar en el nuevo siglo.

Se escogió como línea de investigación El análisis fractal y su relación con los modelos no lineales porque este nuevo paradigma de la ciencia contemporánea ha venido cambiando la manera de ver algunas áreas de la Ingeniería, que por sus dificultades para realizar mediciones y apreciaciones cuantitativas, se habían quedado rezagadas dentro del contexto técnico propio de su saber. 
Entre ellas están el análisis y la observación de los Suelos y las Rocas, el análisis de Señales Sísmicas, problemas de drenaje y movimiento de sedimentos y fluidos, análisis de caudales y la relación entre lluvias y escorrentías, entre otros.

El primer proyecto de la línea se desarrolló conjuntamente con el Instituto Geográfico Agustín Codazzi y se denominó "Estimación de dimensiones fractales para aplicación en física de Suelos". En este proyecto, se consiguió la apropiación de la tecnología para analizar fenómenos desde el punto de vista de los fractales y se adquirieron las herramientas teóricas y de software necesarias para su implementación en nuestro medio.

Actualmente se está desarrollando conjuntamente con el Centro Internacional de Física CIF, el proyecto "Análisis fractal de la línea de base de macrófagos control e infectados con Leishmania Amazonensis" el cual se presentó en el IV Congreso Iberoamericano de Biofísica que se llevó a cabo en Alicante (España). También se presentó ante Colciencias la ampliación del proyecto sobre Leishmaniasis por parte del CIF siendo aprobado. En dicho proyecto la Facultad de Ingeniería de la Universidad Militar Nueva Granada participará como coinvestigadora.

Se puede decir que esta línea está consolidada y podrá apoyar en el futuro a las demás líneas de investigación, permitiendo el objetivo y los logros que se ha propuesto.

En el campo de la didáctica de la física se desarrolló el proyecto: "Diseño de un módulo didáctico para la aprehensión de los conceptos de espacio y masa en ingeniería"

\section{Areas de Geotecnia y Construcción}

La implementación de esta línea se inició con el desarrollo del Proyecto de Investigación " Evaluación de las condiciones de estabilidad del terreno en la zona del ponteadero y de los estribos del puente vial sobre el río Anaime en Cajamarca Tolima, dirigido por el Geólogo Julio Alberto Triviño, Docente de la Facultad, cuyos resultados fueron entregados al Ministerio de Transporte y a la Gobernación del Tolima.

Relacionadas con las áreas de Geotecnia y construcción están en proceso de implementación tres líneas de investigación: en Concreto, en Pavimentos y en Seguridad e Higiene Industrial; esta última incluye la industria de la construcción y están orientadas a desarrollar proyectos que ofrezcan soluciones a diferentes problemas detectados en cada una.

\section{- Línea de Investigación en Concreto}

El concreto es el material de construcción más utilizado para la ejecución de cualquier obra. Debido a su complejidad es necesario diseñar la mezcla según las características mecánicas, físicas y químicas a la cual va estar sometido.

El contacto continuo con este material en la vida cotidiana y la necesidad de eliminar los problemas (patología) que lo aquejan, ha llevado a mejorarlo y a conseguir concretos más acordes con nuestro tiempo. Por tal razón, es muy importante el fomento de la investigación en nuevos diseños que respondan a las necesidades del mundo actual.

\section{- Línea de investigación en Pavimentos}

Mediante la implementación de esta línea, la Facultad de Ingeniería busca desarrollar proyectos de investigación cuyos resultados aporten a la solución de los diferentes problemas de diseño y construcción de estructuras de pavimentos tanto flexibles, como rígidos o articulados, mediante la adición de diferentes materiales y la innovación de procedimientos, con el objeto de lograr reducciones en los costos por el incremento de las propiedades mecánicas, físicas y químicas de los materiales que componen la estructura del pavimento. 


\section{Temas de investigación}

Adición de desperdicio metálico y plástico en estruccuras de pavimenco rígido y flexible.

Utilización de materiales para mejorar y/o estabilizar bases y subbases granulares

- Línea de investigación en Seguridad e Higiene Induscrial.

Al establecer una línea en Seguridad e Higiene Industrial, se busca desarrollar proyectos de investigación que permitan plantear soluciones a los riesgos para la salud de los individuos en su ámbito laboral.

La interdisciplinariedad se aplica en esta línea para la previsión y prevención de los riesgos laborales y/o la solución de problemas detectados dando aplicación a la Seguridad y la Higiene Industrial en la construcción.

Teniendo en cuenta las posibles alteraciones del estado de salud de los trabajadores en su ambiente laboral, el Estado busca que las empresas desarrollen políticas de protección a sus miembros contra los riesgos ocupacionales, mediante la planeación, elaboración, ejecución y evaluación del Programa de Salud Ocupacional cuyo marco juridico abarca leyes, decretos y resoluciones dentro de las que se cuenta la Ley 9 de 1979, Resolución 2400 del 22 de Mayo de 1979. Decreto 614 de 1984, Resolución 2013 de 1986, Resolución 1016 del 31 de Marzo de 1989, Decreto 1295 de 1994, entre otros.

Al considerar la Seguridad y la Higiene Industrial se escimula la producción, se disminuyen las calgas sociales, se enriquece el capital humano y se optimizan los ambientes laborales disminuyendo así misnzo el costo de vida. Esta línea de investigación ha generado el diseño del Programa de Salud Ocupacional para la Universidad Militar Nueva Granada, el cual ha servido de base para generar el desarrollo de otros proyectos de investigación que beneficiarán a la institución.

Temas de investigación y campos de acción:

- Diseño de Programas de Salud Ocupacional según el carácter de cada empresa

- Delección y control de riesgos

- Seguridad e higiene en la construcción

- Prevención de accidentes de trabajo

- Medidas de seguridad y optimización de los ambientes de trabajo

- Diseño y coordinación de programas de capacitación

\section{Area vias, tránsito y transporte}

Esta área de investigación cuenta con el apoyo de un convenio con El Instituto Nacional de Vías y actualmente se está tramitando otro con el Instituto de Desarrollo Urbano (IDU). Se han desarrollado los siguientes trabajos de investigación con la Universidad del Cauca y otras universidades del país, los cuales concaron con asesoría internacional y sus resultados tenidos en cuenta en la actualización del Manual de Capacidad Vial Colombiana para carreteras de dos carriles, jublicado por el Ministerio de transporte en 1996.

- Verificación del estudio: utilización total o parcial de la berma para circulación del trán. sito.

- Análisis de la capacidad de servicio por sentido de circulación

- Factibilidad económica de carriles de adelanto para la vía Bogotá Tunja

- Efecto de las condiciones ambientales en la velocidad promedio del flujo vehicular

\section{Area sanitaria y ambiental}

Se encuentra en proceso de implementación esta importante área de investigación conjuntamente con la Facultad de Biología Aplicada y propenderá por la solución de problemas relacionados con los 
componentes: agua, aire y suelo. Se pretende desarrollar trabajos de investigación tales como:

- Diseño de sistemas de control de la contaminación del agua a la luz de la normatividad vigente en el país, en los municipios, e instalaciones de las Fuerzas Militares. (Batallones, Casas Fiscales, Barrios periféricos, etc.)

- Seguimiento de los sistemas de tratamiento de aguas residuales construidas por la CAR en el área de la Sabana de Bogotá.

- Mejorar y simplificar las técnicas de tratamiento y diseño de plantas para aguas residuales y para aguas de consumo humano

- Conservación de recursos Hídricos

- Estudiar la eficiencia del tanque séptico de filtro percolador anaerobio.

Actualmente se adelanta el proyecto "Identificación y prueba de bioindicadores y recuperadores para la descontaminación del río Bogotá".

\section{Area de mecatrónica}

Se tiene previsto desarrollar líneas de investigación en robótica e inteligencia artificial , sensórica y automatización. Actualmente se desarrolla el proyecto "Diseño de un robot manipulador con fines didácticos para la Facultad de Ingeniería de la Universidad Militar", el cual se ejecutará en dos etapas la de diseño y la de construcción.

\section{Bibliografía}

BRIONES, G. Métodos y Técnicas de Investigación para las Ciencias Sociales. Ed. Trillas, México, 1982

CERDA, Gutiérrez Hugo. Cómo elaborar Proyectos. Ed. Magisterio. Bogotá, sin fecha.

ICFES. SERIE APRENDER A INVESTIGAR. EL PROYECTO DE INVESTIGACIÓN. Módulo 5. Mario Tamayo Tamayo. Ed. Feviva S.A. Cali, Valle. 1995.

UNIVERSIDAD MILITAR "NUEVA GRANADA". Comité Central de Investigaciones. Informes de Avance y Finales de los Diferentes Proyectos Desarrollados por la Facultad de Ingeniería. 1994 - 2000. 
Figura 1

Organización de la Investigación en la Facultad de Ingeniería de la UMNG

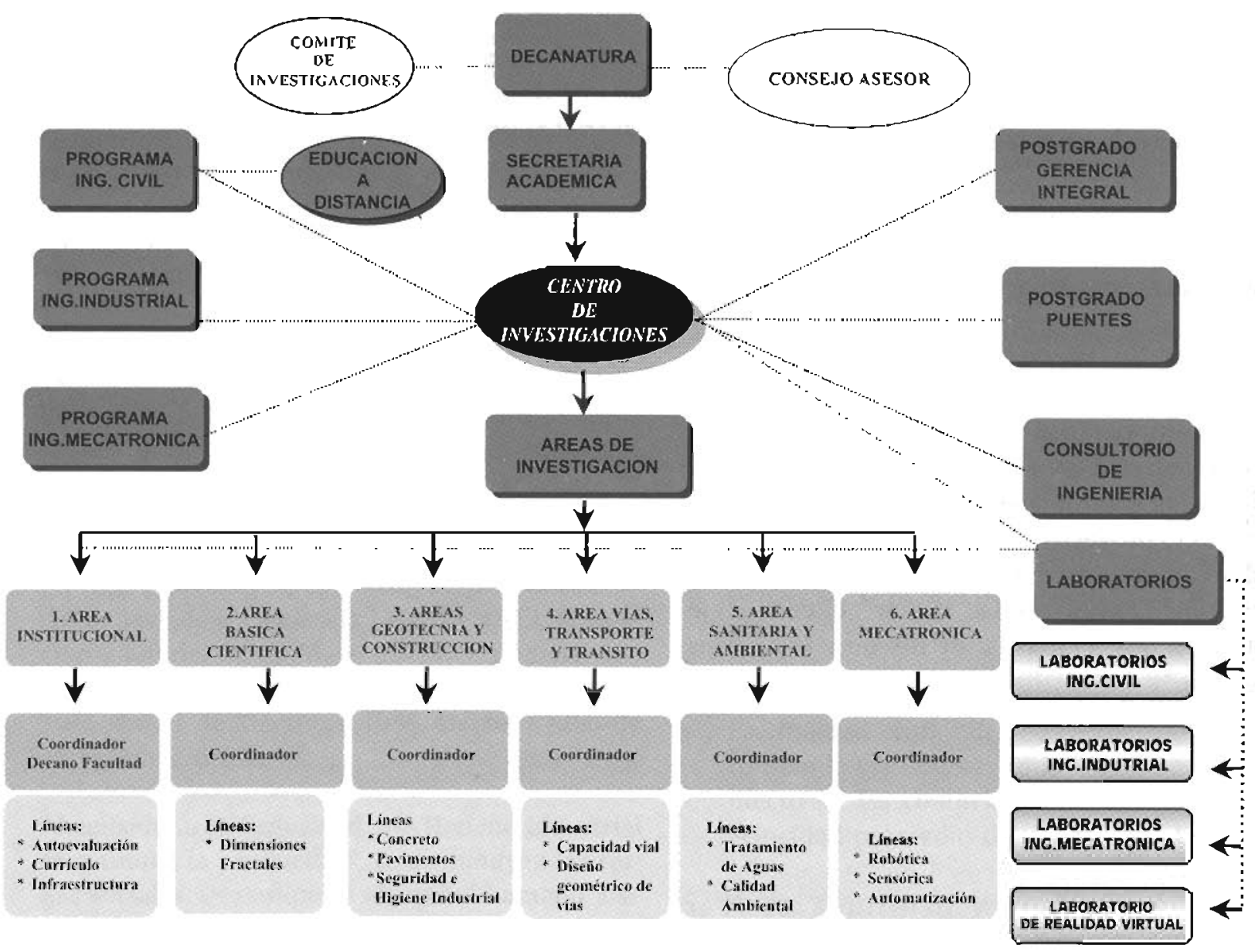

